\title{
Optical characteristics of triclinic rubidium hydrogen selenate
}

\author{
L. Guilbert, J. P. Salvestrini, P. Kolata, F. X. Abrial, and M. D. Fontana \\ Laboratoire Matériaux Optiques à Propriétés Spécifiques, Centre Lorrain d'Optique et d'Electronique des Solides, \\ Université de Metz-Supelec, 2, rue E. Belin, 57078 Metz Cedex 3, France \\ Z. Czapla \\ Institute of Experimental Physics, University of Wroclaw, Pl. Maxa Borna 9, 50-205 Wroclaw, Poland
}

\begin{abstract}
The refractive indices of rubidium hydrogen selenate are measured for several wavelengths at room temperature, and the transmission spectrum is measured from the UV $(240 \mathrm{~nm})$ to the near IR $(2000 \mathrm{~nm})$. The orientation of the optical indicatrix with respect to the crystal axes is also determined at several wavelengths in the visible range. Using a new method based on the deflection of light by the ferroelastic d omain structure, we also determine refined values for birefringence at several w avelengths. Finally, the dispersion of the three birefringences in the range $450-900 \mathrm{~nm}$ is deduced from polarimetric measurements. This set of results yields complete knowledge of the linear optical characteristics required for interpretation of the electro-optical and nonlinear optical properties of this compound.
\end{abstract}

\section{INTRODUCTION}

At room temperature, rubidium hydrogen selenate (RHSe) is in a ferroelectric-ferroelastic phase with the triclinic structure ${ }^{1}$ (space group $P 1$ ). This phase always exhibits a layered domain structure, with domain walls parallel to (001) reticular planes and the spontaneous polarization oriented close to the $b$ axis. $^{2}$ Neighboring domains are symmetrical to each other in a two-fold rotation around the axis perpendicular to the $(a, b)$ planes. This domain structure can be considered especially soft at room temperature, because it can easily be reversed by application of mechanical stresses or electric fields and it reappears spontaneously as soon as the field is released after saturation. This soft ferroelectric behavior is the basic process involved in some interesting properties of RHSe: a pronounced electro-optic effect at low frequency ${ }^{3}$ and electrical modulation of the deflection of light. ${ }^{4}$ However-and probably because of this inherent domain structure- the refractive indices and the ellipsoid orientation of RHSe have not been measured with good accuracy. A better knowledge of the optical properties of this material is essential for its application in electrooptical devices.

In this paper we first determine the following optical characteristics of RHSe at room temperature: the transmission spectrum from the UV $(240 \mathrm{~nm})$ to the IR (2000 $\mathrm{nm}$ ), the orientation of the optical indicatrix relative to the crystal axes for several wavelengths, and the values of two refractive indices measured by Pulfrich refractometry and by Michelson interferometry. The accuracy in measurement of the refractive indices is relatively poor, and the third refractive index has not been determined. Therefore we use the property of light deflection by the domain structure ${ }^{5}$ to obtain refined values of two birefringences at the same wavelengths as for index measurements. An important advantage of this method compared with classical interferometric methods is that the birefringences are directly determined from accurate measurements of the deflection angles and independently from the sample thickness. The latter parameter is not required and therefore does not affect the accuracy in the determination of the birefringence. In a last step, from polarimetric measurements we deduce the overall dispersion for the three birefringences versus wavelength in the range $450-900 \mathrm{~nm}$.

\section{SAMPLE PREPARATION AND DEFINITIONS OF AXES}

Pure $\mathrm{RbHSeO}_{4}$ crystals were grown at $305 \mathrm{~K}$ by evaporation from saturated water solutions. From as-grown single crystals (typically $30 \mathrm{~mm} \times 20 \mathrm{~mm} \times 15 \mathrm{~mm}$ ) parallelepipedic samples and thin plates were cut and polished along the appropriate directions for the experiments. All samples were in the multidomain state, and we did not try to achieve a single-domain state because domains reappear immediately after any treatment. In what follows, any plate will be designated by the axis perpendicular to its optical faces: For instance, $c$ cut means that the faces are cut and polished perpendicular to the $c$ axis and that generally the light is propagated along this $c$ axis. The various cuttings and orientations, denoted $a$, $b$, and $c$, refer to the so-called pseudo-orthorhombic axis system, which is shown in Fig. 1(a) and defined from crystal axes $a_{0}, b_{0}$, and $c_{0}$ of the triclinic lattice as follows: $c$ is the axis perpendicular to the (001) domain walls and is 


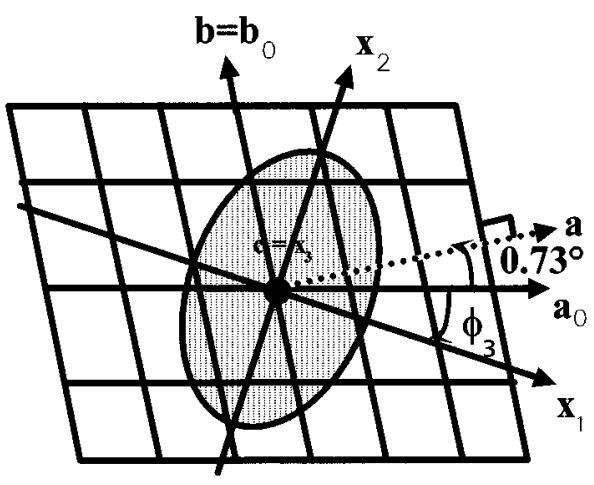

(a)

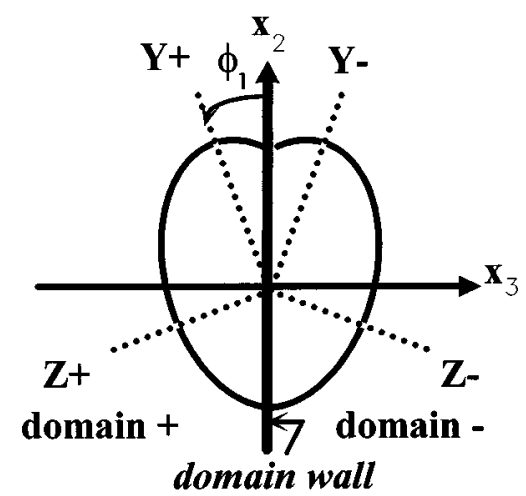

(b)

Fig. 1. Two axis systems. (a) Orthogonal axis system $(a, b, c)$ of the pseudo-orthorhombic structure, and pseudoprincipal axes $\left(x_{1}, x_{2}, x_{3}\right)$ of the average monoclinic structure (see text, Section 2). (b) True principal axes $(X, Y, Z)$ of the triclinic structure in neighboring domains.

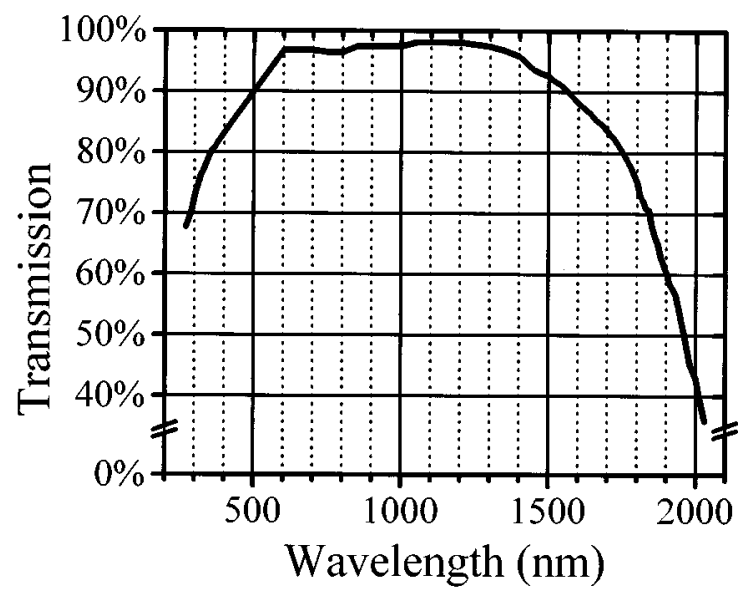

Fig. 2. Transmission spectrum of RHSe for unpolarized light propagating along the $c$ axis and corrected to account for Fresnel losses. (Sample thickness, $3.35 \mathrm{~mm}$.)

oriented in the same way as the pyroelectric $c_{0}$ axis, $b$ $=b_{0}$ is the true crystal axis of the triclinic lattice, and $a$ is the axis perpendicular to $b_{0}$ in the (001) reticular planes.

However, inasmuch as the crystallographic angle $\gamma$ between the $a_{0}$ and $b_{0}$ axes is very close to $90^{\circ}(\gamma$ $\left.=90.73^{\circ}\right)$ the distinction between the $a$ and the $a_{0}$ axes is not significant in most cases.
Another axis system $\left(x_{1}, x_{2}, x_{3}\right)$, which is better suited than the $(a, b, c)$ system to describe the optical properties, is also used below: $x_{1}$ and $x_{2}$ are optical neutral lines (fast and slow, respectively) in the $(a, b)$ plane and $x_{3}=c$ is the axis perpendicular to the $(a, b)$ plane.

These axes should not be confused with the principal dielectric axes $(X, Y, Z)$ of the triclinic, single-domain crystal [Fig. 1(b)]. In a multidomain crystal the axes $x_{1}$, $x_{2}$, and $x_{3}$ correspond to the average positions of the principal axes $(X, Y, Z)$ through the domain structure, so they can be called the pseudoprincipal axes. The corresponding indices $n_{1}, n_{2}$, and $n_{3}$ on the indicatrix are called the pseudoprincipal indices, and the corresponding birefringences $\Delta n_{1}, \Delta n_{2}$, and $\Delta n_{3}$ in the perpendicular planes are called the pseudoprincipal birefringences.

\section{TRANSMISSION SPECTRUM}

The transmission spectrum of RHSe was measured under unpolarized light propagating along the $c$ axis (perpendicular to the domain walls). This configuration was chosen because it is the only one for which the light crosses the crystal without any deflection. For this experiment we used a 3.35-mm-thick, $c$-cut sample and a Cary 17 absorption setup. The results obtained in the range 240$2000 \mathrm{~nm}$ are plotted in Fig. 2. The values are corrected to account for the Fresnel losses. The remaining losses in the transparency range can be attributed to the imperfect quality of the polishing and to the moisture that is due to the relatively large hygroscopy of the crystal. Note that the transparency range on the IR side is wider than that of potassium dihydrogen phosphate (KDP) and similar to that of the deuterated compound, $\mathrm{KD}^{*} \mathrm{P} .{ }^{6}$

\section{ORIENTATION OF THE OPTICAL INDICATRIX}

The ferroelectric-ferroelastic domains of RHSe are clearly visible under a polarizing microscope [Fig. 3(a)] in both $a$-cut and $b$-cut plates. ${ }^{4} \mathrm{As}$ in any ferroelastic crystal, the optical contrast of the domain structure between crossed polarizers is due to the mutual tilt of the optical indicatrices in neighboring domains. This tilt angle $( \pm \phi$ alternately with respect to the domain wall) is schematically illustrated in Fig. 3(b).

However, because angle $\phi$ is small, the measurement under the microscope is not accurate. To refine the measurement of $\phi_{a}$ and $\phi_{b}$, we prepared $a$ - and $b$-cut plates (approximately $1 \mathrm{~mm}$ thick). The sample was placed between two Nicol polarizers. Several wavelengths were used (633, 585, 514, 488, and $458 \mathrm{~nm}$ ) from argon and helium-neon lasers. The beam was carefully focused upon a macro domain (typically 50-200 $\mu \mathrm{m}$ wide) previously observed under the microscope. Extinction was achieved between crossed polarizers; then the beam was shifted to an opposite macro domain and we achieved extinction again by rotating the polarizers. The rotation angle between the two positions has a value of $2|\phi|$, with a typical error of $\pm 0.2^{\circ}$. The results obtained in this way for $\phi_{a}$ and $\phi_{b}$ are listed in Table 1. Note that the results for $\phi_{b}$ are not so accurate and reproducible as those for $\phi_{a}$, for which an explanation is given in Section 8 below. 
(a)

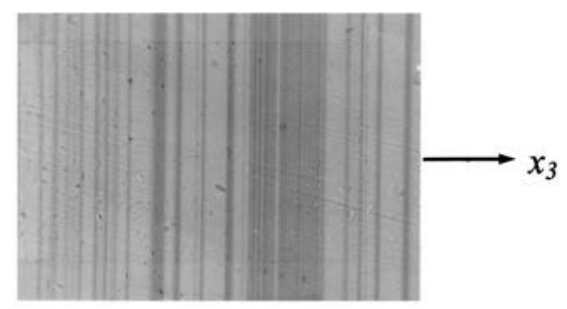

(b)

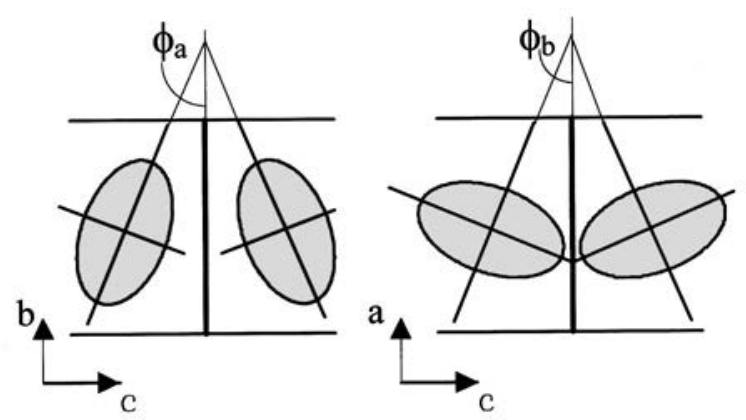

Fig. 3. (a) Domain structure observed under a polarizing microscope in a $b$-cut plate. (b) Tilt angle of the neutral lines in the $(b, c)$ and $(a, c)$ planes.

Table 1. Tilt Angles of the Optical Indicatrix in $\mathbf{R H S e}^{a}$

\begin{tabular}{clcc}
\hline Wavelength $(\mathrm{nm})$ & $\phi_{a}(\mathrm{deg})$ & $\phi_{b}(\mathrm{deg})$ & $\phi_{c}(\mathrm{deg})$ \\
\hline 1321 & $-2.5( \pm 0.2)$ & - & - \\
633 & $-2.25( \pm 0.1)$ & $1.3( \pm 0.4)$ & $-6.0( \pm 0.2)$ \\
514 & $-1.9( \pm 0.15)$ & $1.0( \pm 0.4)$ & - \\
458 & $-1.8( \pm 0.15)$ & $1.0( \pm 0.4)$ & - \\
\hline
\end{tabular}

${ }^{a}$ The value and the sign of each angle refers to the oriented orthogonal system $(a, b, c)$ formed by the pseudo-orthorhombic axes.

We also used a slightly different method to measure $\phi_{a}$ and $\phi_{b}$ : the sample ( $a$ or $b$ cut) was prepared with silver-paste electrodes deposited upon the $b$ faces and subjected to a dc voltage of $250 \mathrm{~V}$, which is usually sufficient to produce a nearly single-domain state. The laser beam was not focused so we could avoid any possibility that a big part of the light could pass through one reverse domain that could remain in the sample despite the electrical saturation. Extinction was achieved at saturation between crossed polarizers; then the dc field was reversed and extinction was achieved again. The values obtained in this way (at $633 \mathrm{~nm}$ ) for $\phi_{a}$ and $\phi_{b}$ were $2.0^{\circ}$ and $1.2^{\circ}$, respectively (values corrected in accounting for the ferroelastic strain at saturation). The value for $\phi_{a}$ is slightly smaller than by the first method, because in the second method the $a$-cut plate was probably not fully saturated. Although it is less accurate, the second method allows us to determine the sign of the angles. Depending on the sign of the electric field, the shear strain of the sample can be observed because of the angular displacement of the deflected beams while the field is reversed. From the knowledge of the triclinic angles $(\alpha$ $\left.>90^{\circ}, \beta<90^{\circ}\right)$ it is easy to determine the signs of $\phi_{a}$ and $\phi_{b}$ relative to the oriented axis system $(a, b, c)$. The values obtained by Tsukamoto ${ }^{4}$ at $633 \mathrm{~nm}$ for $\phi_{a}$ and $\phi_{b}\left(5.5^{\circ}\right.$ and $11.0^{\circ}$, respectively) were much larger than our results. This discrepancy must be attributed to the fact that Tsukamoto used an indirect method, which was based on the extinction of the deflected beams (instead of the direct beam).

The third angle, $\phi_{c}$, defined as the angle between pseudo-orthorhombic axis $a(b)$ and the neutral line $x_{1}$ $\left(x_{2}\right)$ in the $(a, b)$ plane, was measured on a $1 \mathrm{~mm}$-thick $c$-cut plate. Because the $c$ axis is perpendicular to the domain walls, the whole crystal is optically continuous in this direction despite the domain structure, so the extinction position can be directly measured relative to a natural growth face (100) taken as a reference. The value measured at $633 \mathrm{~nm}$ is $\phi_{c}=-6.0^{\circ}\left( \pm 0.2^{\circ}\right)$.

\section{REFRACTIVE INDICES}

The values of the refractive indices were measured by two complementary methods. First we used a Pulfrich refractometer. The parallelepipedic sample used for this experiment was cut perpendicularly to the pseudoprincipal axes $\left(x_{1}, x_{2}, x_{3}\right)$. The $x_{2}$-cut face was aligned horizontally on the prism of the refractometer, and the $x_{3}$-cut face was oriented perpendicularly to the incident beam supplied by a collimated spectral lamp. This configuration allowed us to measure either the $n_{1}$ or the $n_{2}$ index by polarizing the incident light along the horizontal axis or the vertical axis, respectively. The values obtained for three wavelengths of the mercury lamp are listed in Table 2 . By using other configurations we obtained similar results for $n_{1}$ and $n_{2}$. Unfortunately, it was not possible to measure the third index, $n_{3}$, unambiguously with the Pulfrich refractometer because the incident light when it is polarized along $c$ axis is always deflected by the domain structure. This result indicates that the third pseudoprincipal index, $n_{3}$, is smaller than $n_{1}$ and $n_{2}$.

For index measurements we also used an interferometric $\operatorname{method}^{7}$ : The shift of the optical path length through a RHSe plate was measured while the sample was rotated in one arm of a Michelson-type interferometer. From the

Table 2. Pseudoprincipal Refractive Indices of $\mathbf{R H S e}^{a}$

\begin{tabular}{cccc}
\hline & & \multicolumn{2}{c}{ Refractive Index } \\
\cline { 3 - 4 } Wavelength $(\mathrm{nm})$ & $\begin{array}{c}\text { Measurement } \\
\text { Method }\end{array}$ & $n_{1}$ & $n_{2}$ \\
\hline 436 & PR & $1.532(2)$ & $1.579(2)$ \\
546 & PR & $1.526(3)$ & $1.574(3)$ \\
578 & PR & $1.525(3)$ & $1.573(3)$ \\
633 & MI & $1.521(1)$ & $1.563(1)$ \\
1152 & MI & $1.513(1)$ & $1.545(1)$ \\
\hline
\end{tabular}

${ }^{a}$ Measurements at $293 \mathrm{~K}$ by Pulfrich refractometry (PR) and by Michelson interferometry (MI). The accuracy of the PR results is not good because the surface of the crystal was rapidly damaged by the liquid, ensuring contact between the sample and the prism. For MI results, the error in the refractive indices is due mainly to the error in the sample thickness $( \pm 0.15 \%)$ 
positions of the maxima (or minima) on the interferogram it is possible to deduce the index value, provided that the thickness of the sample is perfectly known. We performed the experiment on a $c$-cut RHSe sample (thickness, $2990 \pm 5 \mu \mathrm{m}$ ), using two different wavelengths from helium-neon lasers (633 and $1152 \mathrm{~nm}$ ). These data are also listed in Table 2.

\section{DEFLECTION MEASUREMENTS}

The deflection of light by ferroelastic domain walls can be used to determine the birefringence of a crystal with good accuracy, provided that at least one refractive index is known approximately. This method is rarely employed, and we explain it below. First we describe the deflection phenomenon.

\section{A. Deflection Phenomenon}

As was shown by Tsukamoto and Futama, ${ }^{5}$ the tilt of the optical indicatrix relative to ferroelastic domain walls leads to a deflection phenomenon of the light. Deflection is caused by reflection and refraction processes at the domain walls from high index to low index or vice versa. All possible deflection processes are shown in Fig. 4. An unpolarized incident beam gives rise-as a rule, in the most general case-to six beams, denoted $\mathrm{D}, \mathrm{R}, \mathrm{A}, \mathrm{A}^{\prime}, \mathrm{B}$, and $\mathrm{B}^{\prime}$, at the output of the crystal. Beams $\mathrm{A}, \mathrm{A}^{\prime}$, symmetrical to each other with respect to the plane of domain walls, result from refractive transmission and reflection processes acting from low index to high index at domain walls. These two beams are linearly polarized along the high-index direction. Beams B and B' (also symmetrical to each other with respect to the domain wall) result from refractive transmission and reflection processes from high index to low index. So they are polarized along the lowindex direction and appear only for incidence angles larger than a critical value. The direct beam (D) and the reflected beam $(R)$ are due, respectively, to transmission and reflection processes that do not change the magnitude of the refractive index (i.e., high-to-high or low-to-low). The two beams D and R (symmetrical to each other) are

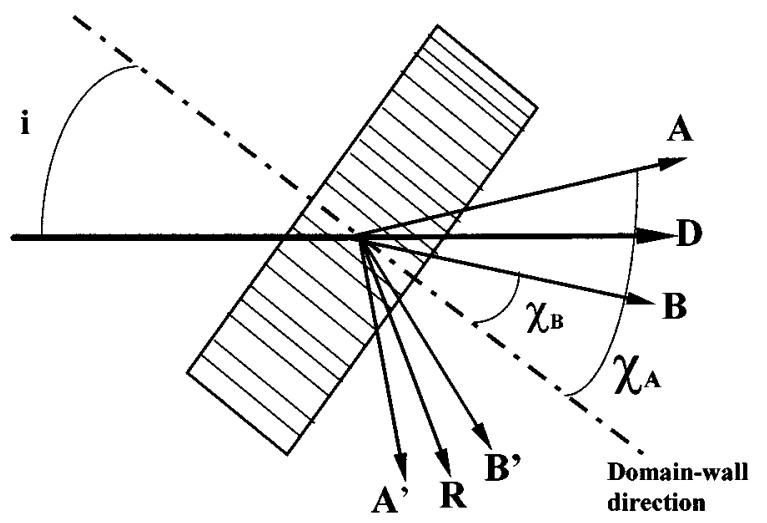

Fig. 4. Deflection processes at domain walls in RHSe. $\mathbf{A}, \mathbf{A}^{\prime}$, refractive transmission and reflection from low index to high in$\operatorname{dex} ; \mathbf{B}, \mathbf{B}^{\prime}$, refractive transmission and reflection from high index to low index. $\mathbf{D}, \mathbf{R}$, nonrefractive transmission and reflection. only slightly refracted at the domain wall (the deviation is negligible) and are both unpolarized.

For a given deflected beam the deflection angle $\chi$ depends not only on the incidence angle $i$ but also on the direction of the cutting axis chosen in the $(a, b)$ plane. As we shall see below, accurate measurements of the deflection angles $\chi$ make it possible to deduce the values of the birefringences.

\section{B. Experimental Results}

Several deflecting RHSe plates, less than $1 \mathrm{~mm}$ thick, were cut perpendicularly to the domain structure: $a$-cut (100), $b$-cut (010), (1 10$)$-cut, $x_{1}$-cut, and $x_{2}$-cut, and two other plates were cut at $-17^{\circ}$ and $+13^{\circ}$ from the $x_{2}$ axis. We recall that $x_{1}$ and $x_{2}$ are defined as the neutral lines in the $(a, b)$ plane and are rotated by $6^{\circ}$ from the $a$ and $b$ axes, respectively. These axes $\left(x_{1}, x_{2}\right)$ are of particular interest for deflection because they correspond to the extremal values (maximal and minimal, respectively) of deflection angle $\chi_{A}$ at zero incidence. Several laser beams were used as a light source, with wavelengths ranging from 454 to $633 \mathrm{~nm}$. The sample was centered on the vertical axis of a rotating holder to vary the angle of incidence $i$. Using a goniometric system, we measured the deflection angles relative to the incident beam, with a typical error of $\pm 0.1^{\circ}$, as a function of the angle of incidence $i$. Complete experiments were made in this way at $633 \mathrm{~nm}$ for the $(100)$ and the $(1 \overline{10})$ plates. The results are plotted in Fig. 5, where the deflection angles have been referred not to the incident beam direction but to the direction of the domain walls (i.e., the angle of incidence $i$ has been subtracted from the measured values to show the symmetry of the deflection phenomenon). As Fig. 5(a) shows, in the case of the (100) plate the $\mathrm{B}-\mathrm{B}^{\prime}$ beams appear above a critical incidence angle $\left(24.5^{\circ}\right)$ that is equal to the deflection angle $\chi_{A}(0)$ of the $\mathrm{A}-\mathrm{A}^{\prime}$ beams at zero incidence. This value is in good agreement with that obtained previously by Tsukamoto. ${ }^{4}$ For the (110) plate [Fig. 5(b)], the value of deflection angle $\chi_{A}(0)$ at zero incidence is $13.7^{\circ}$. The results obtained for deflection angle $\chi_{A}$ at zero incidence in the different plates are listed in Table 3. The measurements versus wavelength were carried out with the $x_{1}$-cut and the $x_{2}$-cut plates. The results are given in Table 4.

\section{Refinement of Birefringence from Deflection Measurements}

As was shown by Tsukamoto et al., ${ }^{8}$ the deflection angles can be calculated from the laws of crystal optics, in any configuration of the incident beam, provided that the following five optical parameters of the deflecting crystal are known: the two tilt angles $\phi_{1}$ and $\phi_{2}$ of the indicatrix relatively to domain walls (as seen from the $x_{1}$ and the $x_{2}$ axes, respectively), or otherwise the two inclination angles $\phi_{a}$ and $\phi_{b}$ (as seen from the $a$ and the $b$ axes, respectively) and the three principal indices $n_{x}, n_{y}$, and $n_{z}$ (or otherwise the pseudoprincipal indices $n_{1}, n_{2}$, and $n_{3}$ ).

However, the deflection angle is sensitive mainly to the birefringence of the plate but is less sensitive to the absolute values of the refractive indices or to the tilt angles of the indicatrix (provided that these angles are small). For instance, in an $x_{1}$-cut plate the deflection angle $\chi_{A}(0)$ at 


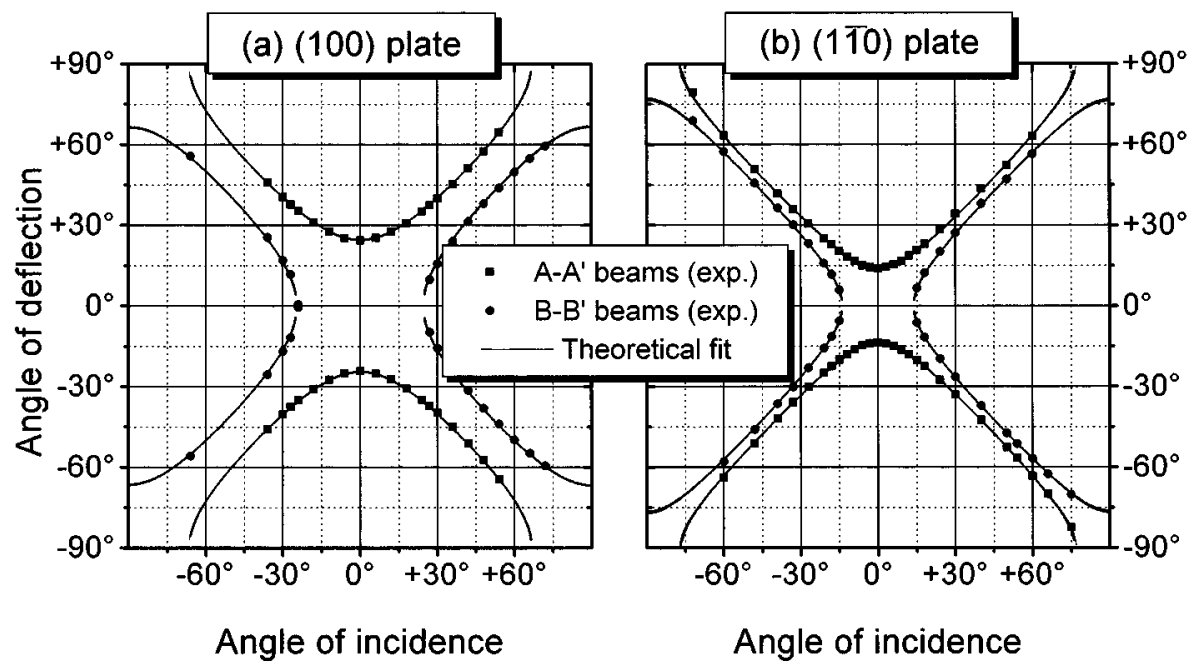

Fig. 5. Deflection angles of beams A and B at $633 \mathrm{~nm}$ as functions of the angle of incidence. The least-squares values of the birefringence taken for the theoretical fitting are $\Delta n_{1}=0.0560$ and $\Delta n_{2}=0.0139$. Other optical parameters involved in the calculation are $n_{2}=1.5632, \phi_{1}=-2.2^{\circ}, \phi_{2}=+0.6^{\circ}, \phi_{3}=-6.0^{\circ}$.

Table 3. Deflection Angle $\chi_{A}(0)$ at Zero Incidence ${ }^{a}$

\begin{tabular}{lcc}
\hline $\begin{array}{c}\text { Cutting Axis } x \\
\text { of the Plate }\end{array}$ & $\begin{array}{c}\text { Position of } x \\
\text { Relative to } x_{1}(\mathrm{deg})\end{array}$ & $\begin{array}{c}\text { Angle of Deflection } \\
\text { at Zero Incidence (deg) }\end{array}$ \\
\hline$x_{1}$ & 0 & 24.6 \\
$a$ & 6 & 24.5 \\
$x_{2}-17^{\circ}$ & 73 & 12.6 \\
$x_{2}$ & 90 & 11.85 \\
$b$ & 96 & 11.9 \\
$x_{2}+13^{\circ}$ & 103 & 13.2 \\
$(1 \overline{1} 0)$ & 110 & 13.7 \\
\hline
\end{tabular}

${ }^{a}$ Measurements at $633 \mathrm{~nm}$ for different plates cut perpendicularly to the domain walls.

Table 4. Deflection Angle $\chi_{A}(0)$ at Zero Incidence As a Function of Wavelength for $x_{1}$-Cut and $x_{2}$-Cut Plates

\begin{tabular}{ccc}
\hline & \multicolumn{2}{c}{ Angle of Deflection at Zero Incidence (deg) } \\
\cline { 2 - 3 } Wavelength $(\mathrm{nm})$ & $x_{1}$-Cut Plate & $x_{2}$-Cut Plate \\
\hline 1321 & $21.4 \pm 0.3$ & - \\
1064 & $22.5 \pm 0.3$ & - \\
820 & $23.7 \pm 0.3$ & - \\
633 & $24.6 \pm 0.1$ & $11.9 \pm 0.1$ \\
514 & $25.4 \pm 0.1$ & $12.1 \pm 0.1$ \\
488 & $25.6 \pm 0.1$ & $12.15 \pm 0.1$ \\
458 & $25.9 \pm 0.1$ & $12.25 \pm 0.1$ \\
\hline
\end{tabular}

zero incidence depends mainly on birefringence $\Delta n_{1}$ in the plane perpendicular to the $x_{1}$ axis. Therefore, from the results of deflection measurements given above, it is possible to refine the values of the two pseudoprincipal birefringences $\Delta n_{1}$ and $\Delta n_{2}$, provided that at least one refractive index is known.

It should be pointed out that within this technique the determination of the birefringence is absolutely indepen- dent of sample thickness $L$. Comparatively, the classical techniques based on interferometry or on polarimetry require $L$ as a parameter in the fitting procedure for determination of refractive index $n$ (or of birefringence $\Delta n$ ): Although the accuracy of the product $L \times(n-1)$ or $L$ $\times \Delta n$ is usually very good (typical relative error is to $10^{-5}$ or $10^{-6}$ ), the final accuracy of the birefringence can be much affected by the inaccuracy of the sample thickness itself (typical relative error $10^{-3}$ to $10^{-4}$ ). Additionally, in our deflection method the sign of each birefringence is determined immediately from the angular position of the input polarizer, ensuring the extinction of the $\mathrm{A}-\mathrm{A}^{\prime}$ deflected beams.

To test the validity of our fitting computations we started from previous studies by Tsukamoto et al. ${ }^{8}$ of other deflecting crystals (Rochelle salt, gadolinium molybdate, bismuth titanium oxide), because their birefringences are well known. We verified that our computations of deflection angles versus incidence were in perfect agreement with the results obtained by Tsukamoto et al. for these crystals. Then we used the same theoretical calculation to fit our own experimental data obtained for RHSe crystals; the fitting parameters were the pseudoprincipal birefringences $\Delta n_{1}$ and $\Delta n_{2}$. One of the three pseudoprincipal indices, $n_{2}$, was assumed to be known: The value obtained previously by Michelson interferometry (see Section 5), $n_{2}=1.5632(633 \mathrm{~nm})$, was chosen.

Complete fits of all deflected beams versus incidence angle have been made with our computer program for (100) and (11 0$)$ plates at $633 \mathrm{~nm}$. The results are plotted in Figs. 5(a) and 5(b), respectively. As can be seen, good agreement is achieved with the experimental data for both plates in the whole range of the incidence angle. The corresponding least-squares values for the pseudoprincipal birefringences at $633 \mathrm{~nm}$ are $\Delta n_{1}$ $=0.0560(2)$ and $\Delta n_{2}=0.0139(1)$. For other wavelengths the birefringences are deduced from the deflection angles $\chi_{A}(0)$ listed in Table 4 (measured for A-type beams 


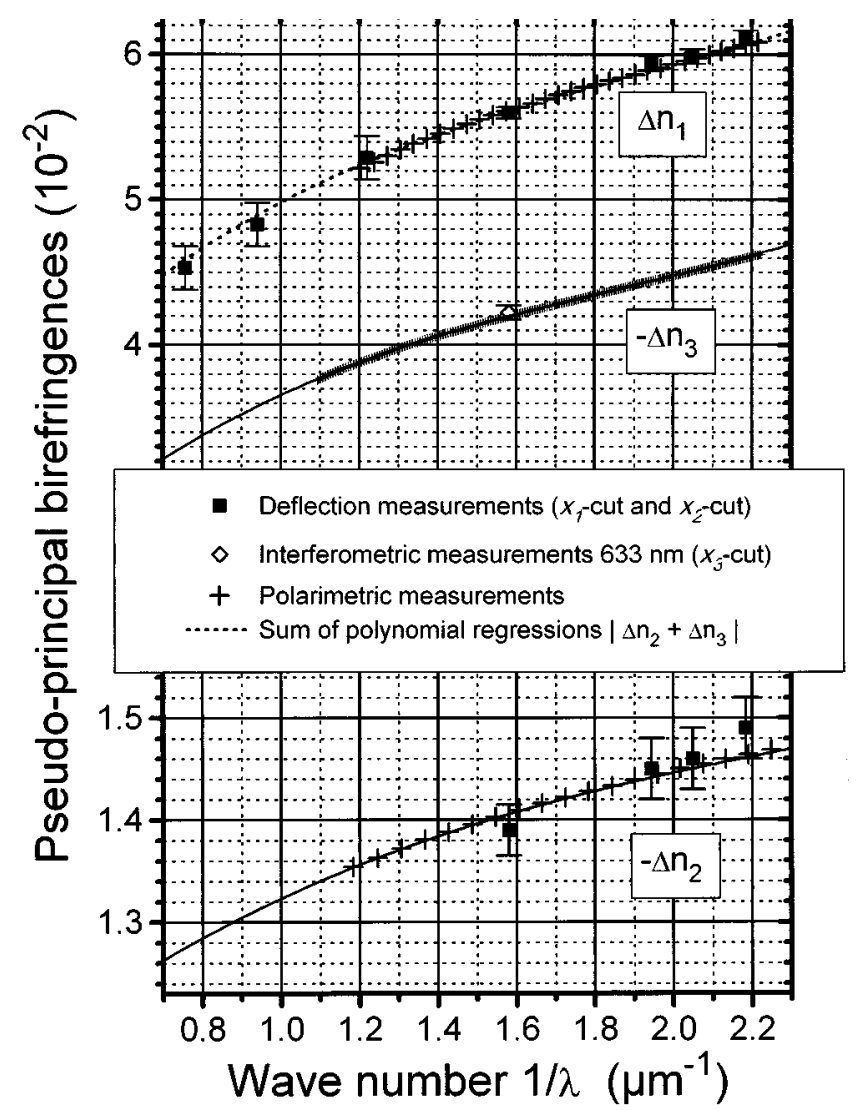

Fig. 6. Dispersion of the pseudoprincipal birefringences deduced from the deflection measurements. The solid curves are polynomial regressions $\Delta n_{2}$ and $\Delta n_{3}$ given by Eqs. (3) and (4) as deduced from polarimetric measurements. $\left|\Delta n_{2}+\Delta n_{3}\right|$ $\approx \Delta n_{1}$.

at zero incidence only). The results are shown in Fig. 6 together with the results deduced from polarimetric measurements (see Section 7 below). The absolute error of birefringences $\Delta n_{1}$ and $\Delta n_{2}$ is deduced from the experimental error on deflection angle $\chi_{A}\left( \pm 0.1^{\circ}\right.$ in the visible range, $\pm 0.3^{\circ}$ in the IR range).

We computed the theoretical variation of deflection angle $\chi_{A}(0)$ at $633 \mathrm{~nm}$ as a function of the propagation direction in the $(a, b)$ plane of the domain walls, using the same parameters as above $\left(\Delta n_{1}=0.0560, \Delta n_{2}\right.$ $\left.=0.0139, n_{2}=1.5632, \phi_{1}=-2.2^{\circ}, \phi_{2}=+0.6^{\circ}\right)$. The curve is drawn in Fig. 7(a) (dashed curve) through the set of experimental data corresponding to the different deflecting plates listed in Table 4 .

\section{BIREFRINGENCE DISPERSIONS BY THE POLARIMETRIC METHOD}

For these experiments a plane-parallel plate is placed between crossed polarizers, both rotated by $45^{\circ}$ from the neutral lines of the plate. As the wavelength is scanned continuously with the aid of a monochromator placed between a white-light source and the sample, an intensity spectrum is recorded (Fig. 8). The maxima correspond to half-wave states of the sample, and the minima correspond to full-wave states. From the positions of these extrema it is possible, in principle, to determine the disper- sion of the birefringence, provided that the sample thickness $L$ is known and that each minimum can be indexed by an integer $m$ ( $m$ is the order of the full-wave state at the corresponding wavelength):

$$
\Delta n(\lambda)=m \lambda / L \quad(m \in \mathscr{N}) .
$$

In practice, indexing the minima without any ambiguity requires at least one value of the birefringence at a particular wavelength. The previous deflection measurements at $633 \mathrm{~nm}$ (Section 6) can provide the requisite results. The $x_{1}$ - and $x_{2}$-cut plates used for this experiment were the same as those described in Section 6. For the $x_{1}$-cut plate (thickness, $430 \pm 5 \mu \mathrm{m}$ ) the 34 minima obtained in the range 900-450 $\mathrm{nm}$ are indexed from 25 \pm 1 to $58 \pm 1$. For the $x_{2}$-cut plate (thickness, 530 $\pm 5 \mu \mathrm{m}$ ) the 10 minima obtained in the same range are indexed unambiguously from 8 to 17 .

For the $x_{3}$-cut plate (thickness, $2990 \pm 5 \mu \mathrm{m}$ ) the indexing of the minima cannot be done from results of deflection measurements, because this configuration gives no deflection. Therefore the indexing is made from the value $\Delta n_{3}$ at $633 \mathrm{~nm}$, which can be deduced from the refractive indices $n_{1}$ and $n_{2}$ measured by Michelson interferometry on the same $x_{3}$-cut sample (see Section 5 ).

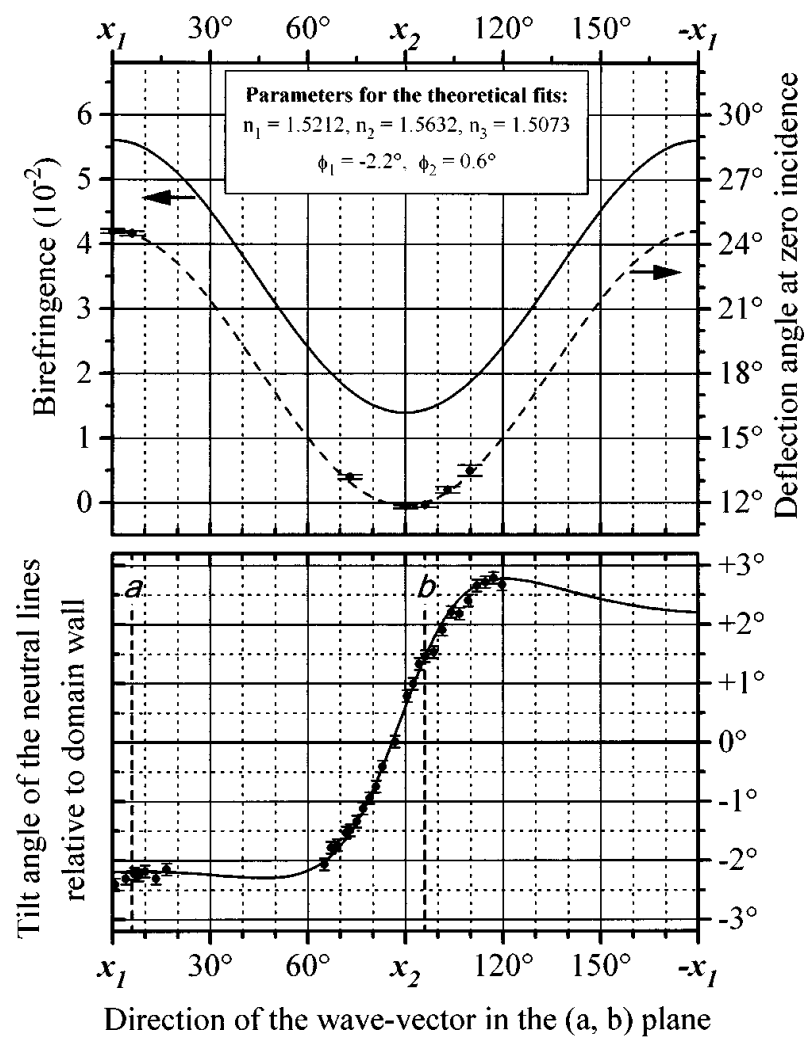

Fig. 7. Variation of the birefringence (solid curve, top figure), the deflection angle (dashed curve, top figure), and the tilt angle of the neutral lines (solid curve, bottom figure) as functions of the propagation direction in the $(a, b)$ plane of domain walls at the wavelength $633 \mathrm{~nm}$. The theoretical curves are calculated from Eqs. (5) and (6) (see text, Section 8). The experimental data (filled circles) are obtained from several deflecting plates cut perpendicularly to the domain walls. 


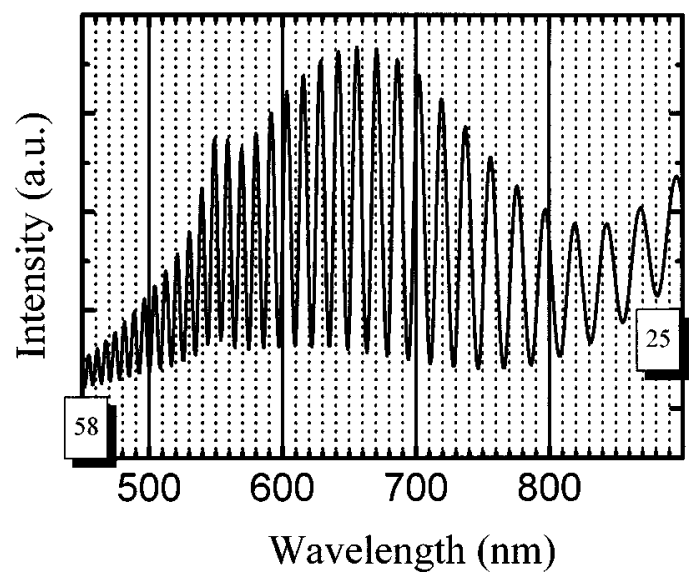

Fig. 8. Intensity spectrum obtained with the $x_{1}$-cut RHSe plate (430 $\mu \mathrm{m}$ thick) between crossed polarizers at $45^{\circ}$ from the neutral lines. The dispersion of the birefringence can be deduced from the spectral positions of the extrema.

This reference point at $633 \mathrm{~nm}$ leads to indexing the 186 minima of the spectrum from $122 \pm 2$ to $307 \pm 2$ in the range $900-450 \mathrm{~nm}$.

The dispersions of the three birefringences $\Delta n_{1}, \Delta n_{2}$, and $\Delta n_{3}$ are then deduced from Eq. (1). The results are plotted in Fig. 6 versus the wave number $1 / \lambda$. The ambiguities that still affect the indexing of the minima $( \pm 1$ for the $x_{1}$-cut plate, \pm 2 for the $x_{3}$-cut plate) are due to experimental errors in the sample thickness $( \pm 5 \mu \mathrm{m})$ and in the birefringence at $633 \mathrm{~nm}$ taken as a reference point $\left( \pm 2 \times 10^{-4}\right)$. These ambiguities do not significantly affect the accuracy of the dispersions. For the $x_{2}$-cut plate there is no ambiguity in the indexing, so the maximal error in the dispersion $\Delta n_{2}\left( \pm 1.3 \times 10^{-4}\right)$ strictly corresponds to the error in the sample thickness.

From these results we can expand the birefringence dispersions by polynomial regressions versus $1 / \lambda$, as follows:

$$
\begin{aligned}
\Delta n_{1}(\lambda)= & 0.01889+0.04719 / \lambda-0.02038 / \lambda^{2} \\
& +0.00345 / \lambda^{3}, \\
-\Delta n_{2}(\lambda)= & 0.01058+0.00368 / \lambda-0.00119 / \lambda^{2} \\
& +0.00016 / \lambda^{3}, \\
-\Delta n_{3}(\lambda)= & 0.01479+0.03367 / \lambda-0.01447 / \lambda^{2} \\
& +0.00257 / \lambda^{3} .
\end{aligned}
$$

The validity of these regressions is restricted to the range of measurements $450-900 \mathrm{~nm}$.

Inasmuch as $\Delta n_{1}, \Delta n_{2}$, and $\Delta n_{3}$ are not principal but are pseudoprincipal birefringences, they should not exactly verify that $\Delta n_{1}+\Delta n_{2}+\Delta n_{3}=0$. Nevertheless, it can be verified from Fig. 6 that the experimental data obtained for $\Delta n_{1}$ are close to the sum of the polynomial regressions $\left|\Delta n_{2}\right|+\left|\Delta n_{3}\right|$ in the whole spectral range. In fact, because the maximum tilt angle of the optical indicatrix is small, one can calculate that the difference should theoretically not exceed $2 \times 10^{-4}$, a value that is below the experimental error.

\section{VARIATIONS OF THE BIREFRINGENCE AND OF THE TILT ANGLE OF THE NEUTRAL LINES VERSUS SAMPLE ORIENTATION}

We show in Fig. 7 the variations of the birefringence, of the deflection angle $\chi_{A}(0)$, and of the tilt angle $\phi$ of the neutral lines with respect to domain walls, as functions of the propagation direction in the plane of domain walls, for the wavelength $633 \mathrm{~nm}$. The variable $x$ corresponds to the angle between the wave vector and pseudoprincipal axis $x_{1}$. Most of the experimental points for tilt angle $\phi$ have been obtained by use of three different plates (perpendicular to $a$, to $x_{2}-17^{\circ}$, and to $x_{2}+13^{\circ}$, respectively) placed between crossed polarizers. Each plate was slightly tilted with respect to the incident beam to vary the propagation direction on each side of the cutting axis. The angle of incidence relative to the cutting axis was limited to $\pm 10^{\circ}$ and corrected to account for the refraction inside the plate. The theoretical variations $\Delta n(x)=n_{+}(x)-n_{-}(x)$ and $\phi(x)$ shown in Fig. 7 are calculated from the laws of crystal optics [Eqs. (5) and (6)] with the same parameters as above $\left(n_{1}=1.5212, n_{2}\right.$ $\left.=1.5632, n_{3}=1.5073, \phi_{1}=-2.2^{\circ}, \phi_{2}=+0.6^{\circ}\right)$ :

$$
\begin{gathered}
\phi(x)=1 / 2 \arctan \left[\frac{b(x)}{a(x)-a_{3}}\right], \\
\frac{1}{{n_{ \pm}}^{2}(x)}=\frac{a(x)+a_{3}}{2} \pm \frac{b(x)}{2 \sin 2 \phi(x)},
\end{gathered}
$$

where

$$
\begin{aligned}
a(x)= & \frac{1}{n_{1}{ }^{2}} \cos ^{2} x+\frac{1}{n_{2}{ }^{2}} \sin ^{2} x, \\
a_{3}= & \frac{1}{n_{3}^{2}}, \\
b(x)= & \left(\frac{1}{n_{2}{ }^{2}}-\frac{1}{n_{3}^{2}}\right) \tan 2 \phi_{1} \cos x \\
& -\left(\frac{1}{n_{3}^{2}}-\frac{1}{n_{1}^{2}}\right) \tan 2 \phi_{2} \sin x .
\end{aligned}
$$

The theoretical variation of deflection angle $\chi_{A}(0, x)$ at zero incidence was also computed and is plotted in Fig. 7(a). The agreement between theoretical and experimental data is fairly good for both tilt angle $\phi$ and deflection angle $\chi_{A}$.

Note that a particular axis exists, close to the $x_{2}$ axis, where tilt angle $\phi$ goes to zero. When the light beam is propagated along this direction, the optical indicatrices in two opposite domains look exactly parallel, and the intensity of the deflected beams weakens drastically. For the same reason, the optical contrast of the domain structure under the polarizing microscope should also vanish, because the multidomain crystal looks optically continuous in this particular direction. This effect is sometimes observed in some slightly misoriented $x_{2}$-cut plates. Moreover, as evidenced in Fig. 7(b), tilt angle $\phi$ varies drastically with the sample orientation in the vicinity of the $x_{2}$ or the $b$ axis, whereas it is almost constant in the vicinity 
of the $x_{1}$ or the $a$ axis. This is certainly the reason why the experimental results in Section 4 were less reproducible for $\phi_{b}$ than for $\phi_{a}$.

\section{SUMMARY}

We have determined the optical characteristics of rubidium hydrogen selenate at room temperature as follows: the transmission spectrum and transparency range, the orientation of the optical indicatrix, the refractive indices, and the dispersion of the birefringences versus wavelength. These data will be particularly important for correct interpretation of the contribution of domain dynamics to the huge electro-optic effect observed in this material at low frequency ${ }^{3,9}$ and for prediction of the dependence of this effect on wavelength and on sample orientation. Moreover, we have emphasized the possibility of and interest in using the light deflection by the domain structure to obtain the birefringence of ferroelastic crystals. This method requires no particular thickness or careful preparation of the plate and can be used directly on multidomain crystals.

\section{ACKNOWLEDGMENTS}

This research was supported by grant 6563 from the Komitet Bodań Naukowych and the Ministère des Affaires Etrangères in the framework of the Polish-French scien- tific exchanges. We are grateful to K. Betzler and B. Tiegel for interferometric measurements of the refractive indices in Osnabrück.

\section{REFERENCES}

1. A. Waskowska, S. Olejnik, K. Lukaszewicz, and T. Glowiak, "Rubidium hydrogen selenate," Acta Crystallogr. Sec. B 34, 3344 (1978).

2. S. Suzuki, T. Osaka, and Y. Makita, "Successive phase transitions in $\mathrm{RbHSeO}_{4}$," J. Phys. Soc. Jpn. 47, 1741 (1979).

3. J. P. Salvestrini, M. D. Fontana, M. Aillerie, and Z. Czapla, "New material with a strong electrooptic effect: rubidium hydrogen selenate," Appl. Phys. Lett. 64, 1920 (1994).

4. T. Tsukamoto, "Deflection of light by ferroelectricferroelastic RbHSeO 4 ," Jpn. J. Appl. Phys. 23, 424 (1984).

5. T. Tsukamoto and H. Futama, "Light deflection induced by ferroelastic layered domains," Phase Transit. 45, 59 (1993).

6. D. Eimerl, "Electrooptic, linear and nonlinear optical properties of KDP and its isomorphs," Ferroelectrics 72, 95 (1987).

7. U. Schlarb and K. Betzler, "Interferometric measurement of refractive indices in $\mathrm{LiNbO}_{3}$," Ferroelectrics 126, 39 (1992).

8. T. Tsukamoto, J. Hatano, and H. Futama, "Deflection of light induced by ferroelectric-ferroelastic crystals," J. Phys. Soc. Jpn. 53, 838 (1984).

9. J. P. Salvestrini, L. Guilbert, and M. Fontana, "Electrooptical properties of rubidium hydrogen selenate: influence of the dc field and origin of the large electro-optic coefficient,” J. Opt. Soc. Am. B 14, 2818 (1997). 